\title{
Un nouvel Asellide cavernicole d'Espagne centrale: Bragasellus lagari n.sp. (Crustacea Isopoda Asellota)
}

\author{
par
}

Jean-Paul HENRY et Guy MAGNIEZ*

La faune d'Asellotes de la péninsule ibérique semble être d'une extrême richesse qualitative. Les Stenasellidae y sont représentés par au moins quatre espèces différentes. Les Asellides proprement dits comprennent à la fois des espèces appartenant à un genre à vaste répartition périméditerranéenne (Proasellus) et aussi des espèces formant des lignées endémiques qui se sont perpétuées dans les réseaux hydrographiques de la péninsule. Ces espèces, anophtalmes pour la plupart, sont aujourd'hui groupées dans les genres Synasellus Braga, 1943; Psammasellus Braga, 1968 (caractérisés en particulier par l'absence du palpe mandibulaire) et Bragasellus Henry et Magniez, 1968 (présentant de nombreux caractères qui les différencient des genres européens. classiques Asellus ou Proasellus).

Jusqu'à présent, les représentants de ces genres endémiques provenaient surtout des régions côtières occidentales de la péninsule, grâce aux multiples prospections et aux belles études de Braga (1942-1967).

Le 21 septembre 1971, un Asellide dépigmenté et anophtalme fut capturé dans la rivière souterraine de la Cueva del Tornero, à Checa, province de Guadalajara (la localité se trouve à environ $150 \mathrm{~km}$ à l'est de Madrid, dans la région des sources du Tage), par notre collègue $M$. Angel Lagar, de Barcelone. Il s'agissait d'une $q$ d'une espèce inconnue, appartenant au genre lusitanien Bragasellus. Une seconde expédition, réalisée le 20 avril 1973, permit de capturer plusieurs autres individus, dont des ot, ce qui rend désormais possible la description de l'espèce. Nous dédions celle-ci à $\mathrm{M}$. Angel Lagar, en le remerciant très vivement pour la confiance qu'il nous témoigne.

Le second lot d'A.sellides de la Cueva del Tornero contenait également un jeune Sténaselle $q$ immature, que nous avons déterminé comme Stenasellus virei Dollfus, 1897, subsp.virei Magniez, 1968, ce qui montre que l'aire de répartition de ce Stenasellidae doit être considérablement étendue vers le Sud.

\section{DESCRIPTION SOMMAIRE DE BRAGASELLUS LAGARI N.SP.:}

Lots capturés: 3 ơ adultes de 7,2; 7,1 et $6 \mathrm{~mm}$; 4qà différents stades de leur cycle génital:

* Laboratoire de Biologie Animale et Générale, Faculté des Sciences de la Vie et de l'Environnement, Université de Dijon, 6, Bd. Gabriel, 21000 DIJON, France. 
*Une 9 à oostégites de repos génital de $8,3 \mathrm{~mm}$,

*Une $\$$ ovigère de 7,9 mm (individu capturé en septembre 1971). Elle porte dans son marsupium 41 embryons segmentés encore contenus dans leur cuticule blastodermique (dimensions approximatives $400 \times 500$ microns). Les organes latéro-dorsaux ou branchies embryonnaires sont bien développés et leur aspect est un peu différent de celui des Asellus ou Proasellus (fig. 2 p).

*Une $\$$ ovigère de $7,4 \mathrm{~mm}$, portant 35 embryons à un stade de développement plus précoce que les précédents (stade de sortie des organes latéro-dorseaux). La taille atteint $350-360$ microns de diamètre. Le vitellus, blanchâtre, est nettement plus abondant que chez les Aselles cavernicoles, mais moins que chez les Sténaselles. * La dernière $q$ était en cours d'intramue lors de sa fixation (la demi-exuvie postérieure avait été abandonnée, mais la partie antérieure du corps conservait son ancienne cuticule). La taille est de $5,8 \mathrm{~mm}$ et les oostégites ne sont pas encore apparents. Tous les individus ont l'intestin rempli d'un limon jaunâtre à grain fin, dont les particules nutritives doivent assurer leur nourriture.

Aspect général: Asellide totalement dépigmenté et anophtalme. La forme générale du corps varie selon l'âge des individus: les 4 plus grands ( $\delta$ de $7,2 \mathrm{~mm}$, $९$ ovigères et $\uparrow$ de $8,3 \mathrm{~mm}$ ), ont un corps très large (coefficient d'allongement de 3 seulement), la largeur maximale étant atteinte au niveau du péréionite $\mathrm{V}(2,5 \mathrm{~mm})$ et le corps se rétrécissant progressivement de part et d'autre (largeur du pléotelson 1,8 mm, pour le $\delta$ de $7,2 \mathrm{~mm}$ ). Les trois derniers individus, plus jeunes, ont un aspect plus élancé (coefficient d'allongement de 4) et les bords du corps sont subparallèles (péréionites IV à VII d'égale largeur: 1,8 $\mathrm{mm}$ pour le ơ de 7,1 $\mathrm{mm}$ ).

Céphalon presque 2 fois plus large que long, avec marge antérieure très légèrement concave; lobes postmandibulaires saillants et limite du somite des maxillipèdes visible. Portions pleurales des péréionites I à VII bien développées et recouvrant largement l'insertion des péréiopodes, dont les coxopodites sont invisibles en vue tergale. L'élargissement du péréion des 4 plus vieux exemplaires est dû à la dilatation encore plus importante des expansions latérales des péréionites. Il s'agit donc d'un caractère de croissance tardive, le corps se développant encore en largeur, alors que la croissance longitudinale est quasi-achevée. Ce grand développement des marges pleurales du péréion se retrouve chez les autres Bragasellus, en particulier chez l'espèce épigée $B$. peltatus, dont la dilatation transversale des péréionites est très remarquable (Braga 1944, figs. 1 et 2). Le caractère de développement des marges pleurales des péréionites, complété par la réduction des coxopodites qui sont ainsi rejetés face ventrale et ankylosés, apparait donc comme typique du genre Bragasellus, de même qu'il l'est du genre américain Lirceus Raf. (=Mancasellus Harger). Chez les Aselles vrais (genres Asellus, Proasellus, etc...), l'adaptation peltastique se traduit en effet, à la fois par l'expansion latérale du péréionite et par celle de la marge externe du coxopodite (épimère ou coxépimère), (Monod 1932). C'est une raison pour voir dans les Bragasellus une lignée indépendante des autres Aselles eurasiatiques.

Chez les plus vieux individus, le pléotelson est subcordiforme; à peu près aussi long que large. Sa largeur, décroissante caudalement, est plus faible que celle des péréionites V, VI et VII $(1,7$ et $2,5 \mathrm{~mm}$, dans le cas du ơ de $7,2 \mathrm{~mm})$ et la pointe 
caudale est bien marquée. Chez les individus plus jeunes, le pléotelson est subquadrangulaire, plus long que large, à bords subparallèles. Sa largeur est sensiblement égale à celle des péréionites et sa pointe caudale est pratiquement inexistante. Dans les deux cas, les pléonites I et II sont étroits et courts, mais bien individualisés (fig. 1A).

\section{Appendices céphaliques}

Antennules plus courtes que la hampe de l'antenne. Hampe de 4 articles et fouet de 6 articles au maximum, avec une lame olfactive de 80 microns sur les 5 distaux. Antennes de $5 \mathrm{~mm}$, pour le of de $7,2 \mathrm{~mm}$; hampe de 6 articles et fouet de 53 articles. Mandibules à corps glabre et très redressé. Apophyse dentaire apicale avec 4 dents, lacinia mobilis terminée par 3 dents. Palpe triarticulé remarquablement développé. Maxillules à lobe interne portant 5 fortes tiges ciliées apicales et à lobe externe muni de 12 tiges distales ainsi que d'une soie lisse sur le bord externe et une autre sur la face sternale. Maxilles trilobées, chaque lobe muni de nombreuses tiges pectinées et falciformes, comme chez B. peltatus.

Maxillipèdes: Aspect typique des Asellidae. Le coxopodite porte un épipodite induré permanent, normalement développé, avec 5 soies lisses à l'angle rostral externe et 3 soies submarginales sur le bord externe. Chez la $q$ ovigère, l'oostégite (Wasserstrudelapparat) est représenté par un lobe ovalaire temporaire, portant une dizaine de tiges ciliées à direction caudale. Ce lobe est donc très semblable à celui des Asellus ou Proasellus. Endite du basipodite portant un rétinacle de 6-8 crochets. Endopodite (palpe) normal de 5 articles.

Péréiopodes: Ils sont relativement grêles et fragiles, mais leur longueur est normale par rapport à celle du corps. Pour le ơ de $7,1 \mathrm{~mm}$, leurs longueurs respectives sont, en millimètres: $1,86-2,92-3,03-3,06-3,41-3,72-3,92$. Leurs longueurs relatives, en prenant le péréiopode II comme référence sont: 63 $100-103-104-116-126-134$. La croissance en longueur des péréiopodes postérieure reste donc très modeste.

Péréiopode $\mathrm{I}$ ơ à propodite allongé, à peine renflé; son bord sternal avec 2 phanères ensiformes insérées au tiers proximal et 5 soies ensiformes submarginales assez espacées sur les deux tiers distaux. Quelques écailles dentées au milieu du bord sternal. Dactylopodite avec 4 épines sternales, dont la plus distale est 2 fois plus longue que les autres. Le propodite du péréiopode I est encore plus faible chez la $q$ et les armatures de propodite et du dactylopodite plus réduites.

Les dactylopodites des péréiopodes II à VII ne portent qu'une seule épine sternale très allongée.

Le péréiopode IV ơ n'est ni raccourci ni déformé en crochet nuptial destiné à maintenir la $\nmid$ lors de l'accouplement. On sait que ce caractère sexuel $\delta$ permanent est bien marqué chez les Asellides épigés, mais qu'il se réduit ou disparaît même chez les espèces adaptées à la vie souterraine. Remarquons que le crochet nuptial est bien développé chez deux espèces de Bragasellus (B. peltatus et B. conimbricensis); ailleurs, il est pratiquement nul. Chez $B$. pauloae, le péréiopode IV est normal, mais c'est le péréiopode $\mathrm{V}$ qui est transformé en crochet nuptial et ce cas est tout à fait exceptionnel. 


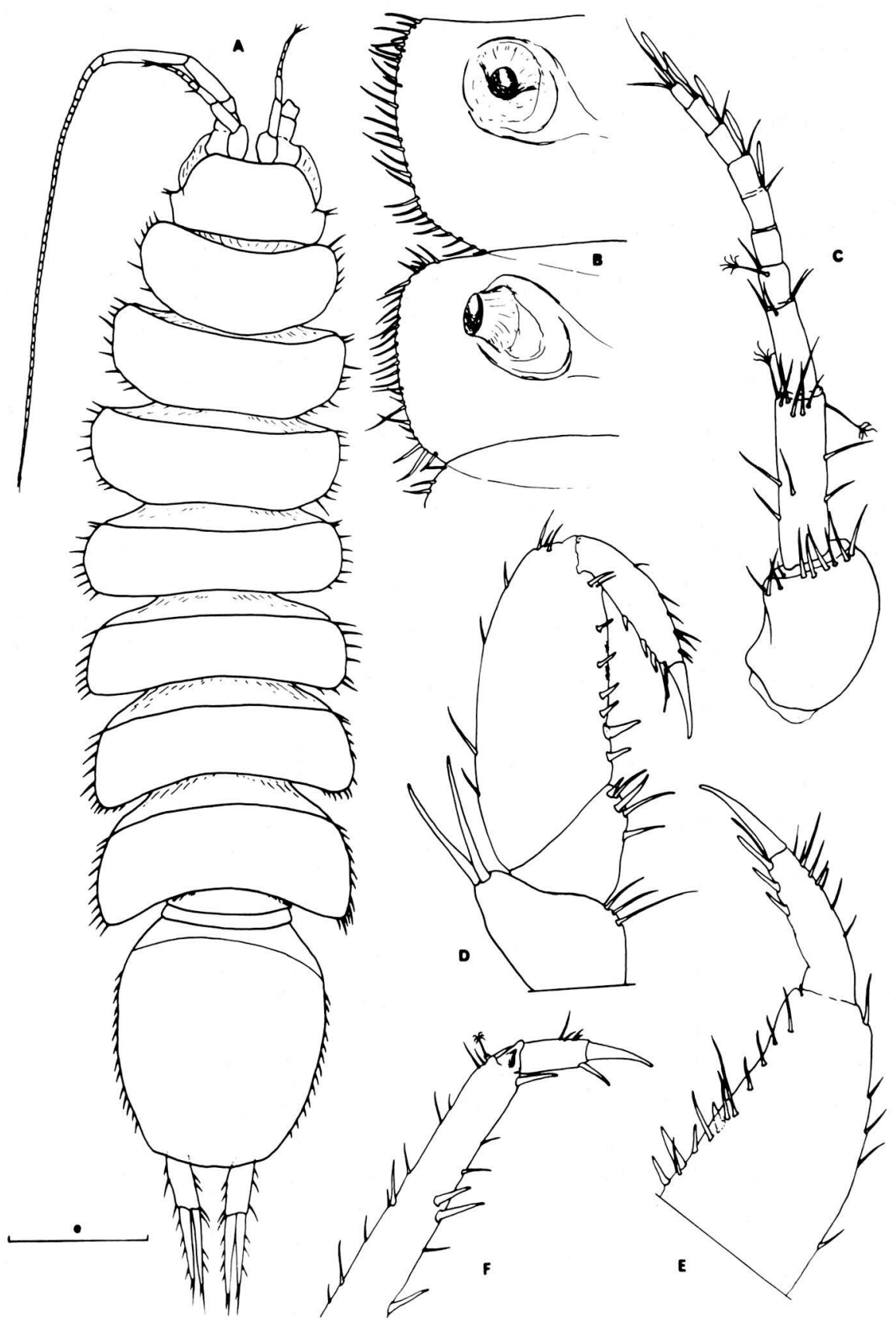


Comme chez $B$ peltatus, les coxopodites des péréiopodes sont très réduits ankylosés et ne forment pas d'épimères. Par leur faible développement, ils rappellent ceux des Lirceus américains. Les coxopodites des péréiopodes I - IV de la $q$ adulte au repos génital portent les oostégites non fonctionnels sous forme de lamelles subquadrangulaires assez longues $(0,4$ à $0,5 \mathrm{~mm}$, pour les II et III qui atteignent leurs symétriques).

Pléopodes I o: Protopodite subtrapézoïdal plus long que large. Bord externe très convexe et bord interne rectiligne avec un seul crochet rétinaculaire assez faible, à insertion très proximale. Quelques écailles épicuticulaires dentées sur la face sternale, près de l'angle distal externe. Exopodite ovalaire, deux fois plus long que large avec deux petites soies lisses près de l'angle proximal interne et une soie lisse inconstante, plus longue, vers le milieu du bord interne. Une série continue de $8-9$ tiges lisses borde le tiers distal de la marge externe, suivie à l'apex de la rame d'un groupe de $6-7$ tiges lisses sur deux rangs. Présence remarquable, sur la face sternale de l'exopodite, d'un renforcement chitineux longitudinal, comme chez certains Conasellus américains, (fig. $2 \mathrm{~K}$ ).

Pléopodes II o: Protopodite subrectangulaire 1 fois $2 / 3$ plus long que large, à bord externe rectiligne et à bord interne régulièrement convexe, avec une soie lisse au milieu. Exopodite biarticulé, à peine plus court que l'endopodite. Article proximal court, portant 1 soie lisse sur la marge externe. Article distal ovalaire, presque 2 fois $1 / 2$ plus long que le proximal, avec 1 soie lisse au milieu du bord externe et 6 soies lisses en groupe sur la partie distale arrondie. Endopodite subcylindrique. Portion proximale très dilatée, portant deux apophyses: l'une interne, cylindro-conique à direction tergale, l'autre externe, aplatie et très saillante, formant un angle aigu avec le corps de la rame. Extrémité de l'endopodite arrondie, sans apophyse tergale, comme chez les Proasellus, prolongée par un tube à direction distale rappelant la "canule" des Conasellus américains. Vésicule interne et fente sternale bien visibles (fig. $2 \mathrm{~N}$ ).

Pléopodes II q: Larnelles triangulaires très allongées, contiguës par leurs bords internes rectilignes, mais sans rétinacle. Leur pointe est occupée par un groupe de soies lisses, comme chez les autres Bragasellus (fig. $3 \mathrm{~S}$ ). Toutefois, chez les ? ovigères, ces appendices prennent une forme différente, subissant un rétrécissement et semblant tronqués distalement en diagonale. Il semble s'agir d'un caractère sexuel $\$$ temporaire (fig. $3 \mathrm{~T}$ ).

Pléopodes III des deux sexes: Protopodite petit et uniarticulé. Allongement de l'exopodite (=opercule) légèrement supérieur à 1,5 . Une suture transversale le divise en deux articles, le proximal étant plus court. Marges externe, distale et

\section{PLANCHE 1.}

A-Bragasellus lagari $\mathrm{n} . \mathrm{sp}$. of de 7,1 $\mathrm{mm}$ en vue tergale; $\mathrm{e}=1 \mathrm{~mm}$.

B-Région pleurale droite des péréionites II et III, face sternale, du o de $7,2 \mathrm{~mm}$; e $=400$ microns.

C-Antennule gauche du précédent; e $=200$ microns.

D-Péréiopode I droit du of de $7,1 \mathrm{~mm}$; e $=200$ microns.

E-Propodite et dactylopodite du péréiopode I de la $\$$ de $7,9 \mathrm{~mm} ; \mathrm{e}=200$ microns.

F-Propodite et dactylopodite du péréiopode IV gauche du ơ de 7,1 mm; e = 200 microns. 


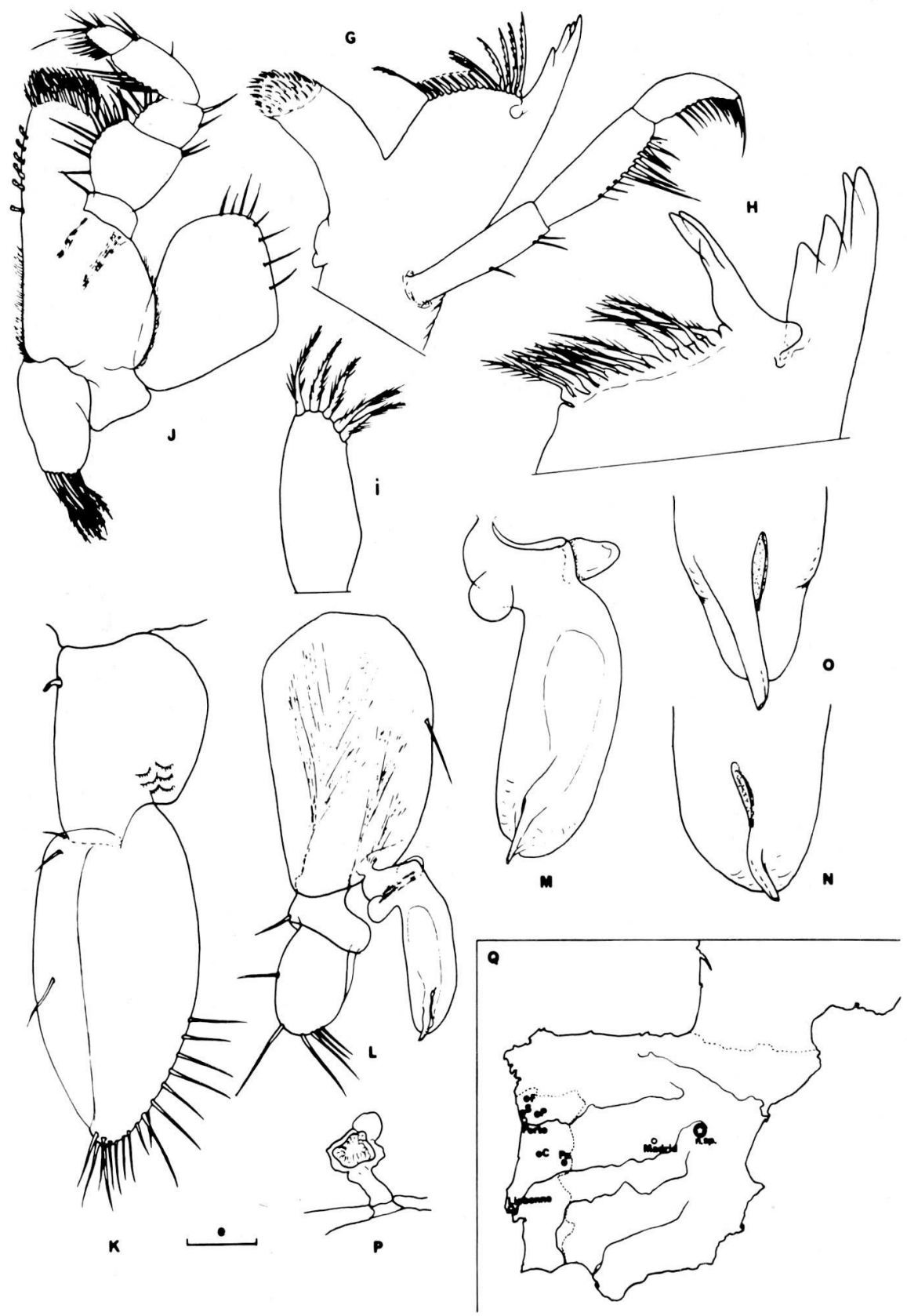


distale-interne portant une série continue de 35 tiges courtes et lisses, certaines submarginales. Deux tiges courtes plus robustes près du bord interne de l'article proximal. Endopodite court, à article distal ovalaire charnu et respiratoire.

Pléopodes IV des deux sexes: Protopodite petit et uniarticulé. Exopodite ovalaire, 1,7 fois plus long que large, divisé en deux articles par une suture oblique (linea area). Bordure ciliee sur la marge externe de l'article proximal. Article distal charnu respiratoire. Endopodite bien développé, à marge externe de l'article proximal finement ciliée. Article distal deux fois plus long que large, charnu et respiratoire. Pléopodes $V$ des deux sexes: Protopodite très petit. Exopodite subovalaire avec article proximal réduit et glabre. Article distal charnu respiratoire. Endopodite aussi long que l'exopodite, mais presque deux fois moins large. Article proximal court et article distal charnu respiratoire.

Uropodes: Leur longueur n'atteint que la moitié de celle du pléotelson. Protopodite assez large, plus court que les deux rames. Endopodite un peu plus long que l'exopodite. Armature de soies bien développée.

Position systématique: Par de nombreux caractères (forme des péréionites et des coxopodites, structure des pléopodes I ó, II $q$ et surtout de l'endopodite des

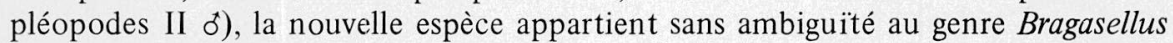
que nous avons défini antérieurement, montrant ainsi a posteriori le bien-fondé de la création de cette entité systématique, dont d'autres espèces cavernicoles ont par ailleurs été découvertes dans le quart Nord-Ouest de l'Espagne et sont en cours d'étude (Ortiz, in litt.).

Remarques biogéographiques: Le genre Bragasellus présente un grand intérêt, qui ira croissant lorsque le nombre des espèces qui le composent, leur écologie, leur biologie et leur répartition seront mieux connus. Cet intérêt est suscité par les raisons suivantes:

1. Il s'agit d'une lignée homogène d'Asellides, bien distincte des autres genres qui ont colonisé les eaux douces européennes ou africaines (genres Asellus et Proasellus) et son origine est certainement différente (souche marine distincte?).

2. Ce genre endémique s'est installé sur un territoire bien délimité (péninsule ibérique), relativement isolé des terres européennes, ce territoire n'ayant pas été

\section{PLANCHE 2.}

G.Bragasellus lagari n. sp., of de 7,2 mm: portion distale de la mandibule droite; $=100$ microns.

H-Portion distale de la mandibule gauche du même; $\mathrm{e}=50$ microns.

I-Portion distale de la lame interne d'une maxillule du même; $\mathrm{e}=100$ microns.

$\mathrm{J}$-Maxillipède de la $\%$ ovigère de $7,4 \mathrm{~mm} ; \mathrm{e}=200$ microns.

K-Pléopode I gauche, face sternale, du o 7,2 mm; e $=100$ microns.

L-Pléopode II droit, face sternale, du ơ de $7,1 \mathrm{~mm}$; e $=100$ microns.

M-Endopodite de pléopode II du ơ de 7,2 mm; e = 50 microns.

N-Portion distale du même; $\mathrm{e}=25$ microns.

O-Portion distale de l'end opodite II ơ de Bragasellus seabrai, d'après Braga, 1943.

P-Un organe latéro-dorsal (branchie embryonnaire) d'un embryon de B. lagari n.sp.; e $=100$ microns.

Q-Carte schématique de la péninsule ibérique, avec les stations des différentes espèces de Bragasellus: $\mathrm{F}=B$. frontellum $; \mathrm{P}=B$. peltatus; $\mathrm{S}=$ B. seabrai $; \mathrm{C}=B$. conimbricensis $; \mathrm{Pa}=B$. pauloae; l'étoile indique la station de $B$. lagari n.sp. 


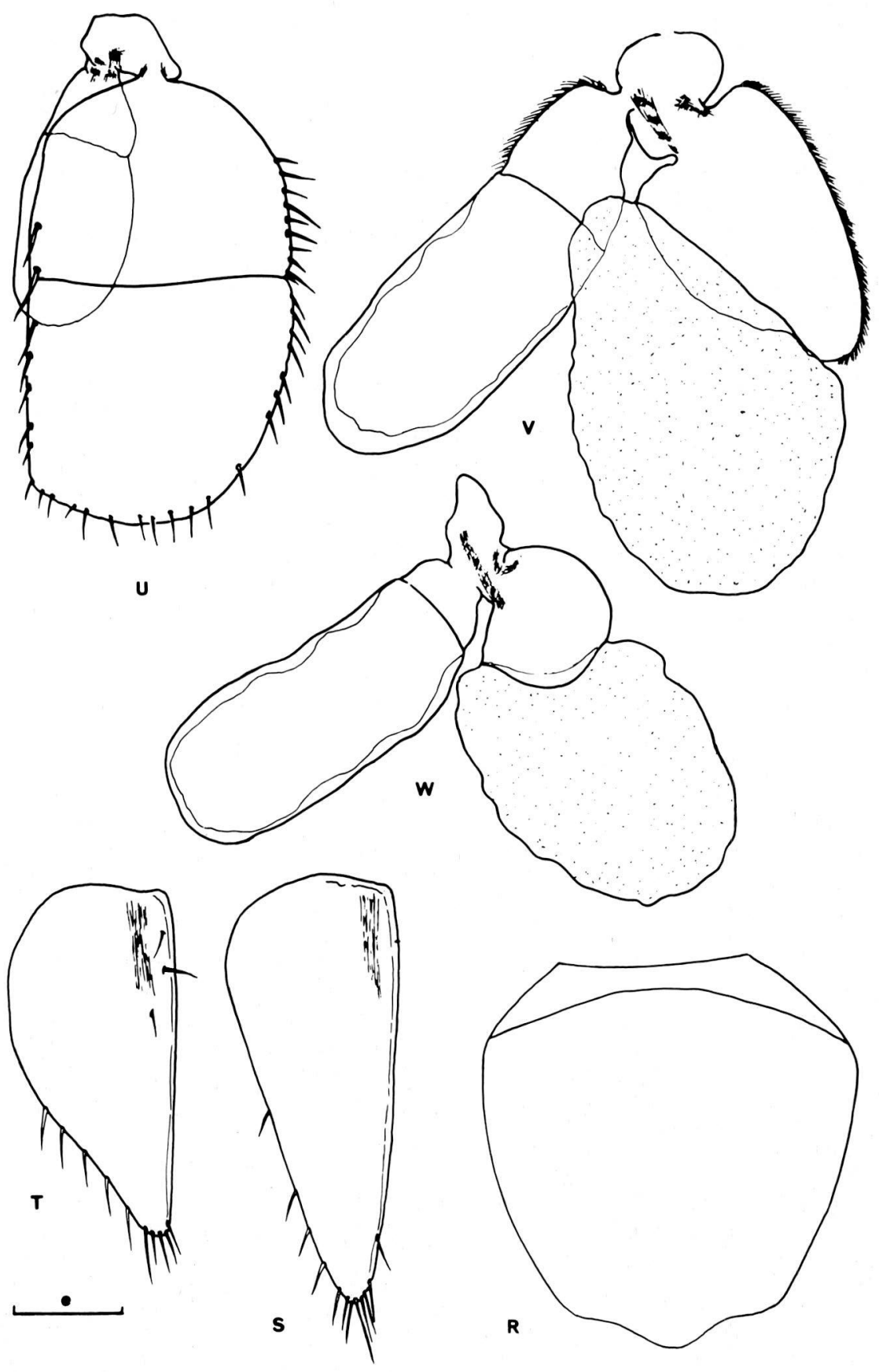


colonisé par les espèces expansives du genre eurasiatique Asellus, comme c'est le cas pour le reste de l'Europe. Les Bragasellus ont donc été tenus à l'abri de ces formes concurrentes et ont pu évoluer dans les eaux douces de ce territoire privilégié. L'histoire de ce peuplement sera vraisemblablement plus simple à reconstituer que celle des peuplements d'Asellides de l'Europe moyenne.

3. Le genre comporte, pour le moment, une forme épigée (pigmentée-oculée) et plusieurs espèces souterraines, soit interstitielles, soit karstiques, dépigmentées et anophtalmes, qui sont manifestement dérivées de la précédente. Il pourrait donc nous apporter des renseignements sur les mécanismes évolutifs qui ont présidé à l'adaptation à la vie souterraine et à la spéciation en milieu dulçaquicole à des époques géologiques récentes.

Il apparaît de plus en plus vraisemblable que les faunes actuelles d'Asellides d'eau douce sont issues de plusieurs centres de dispersion, relativement bien délimites et peu nombreux: Extrême-Orient boréal (Japon et terres voisines), pour les genres Asellus rı.def., Nipponasellus et Uenasellus; Lac Baikal pour Baicalasellus: péninsule balkanique pour Proasellus et Stygasellus; péninsule ibérique pour Bragasellus et Synasellus et enfin Est des Etats-Unis pour Conasellus et Lirceus, ce qui suggère une origine marine pacifique pour les premiers genres, mésogéenne pour Proasellus et atlantique pour les genres lusitaniens et américains.

Manuscrit terminé le 12 juillet 1973

\section{RESUME}

Description d'un nouvel Asellidae apigmenté et anophtalme, appartenant au genre ibérique Bragasellus Henry et Magniez, 1968.

La nouvelle espèce provient de la rivière souterraine de la Cueva del Tornero (Checa, province de Guadalajara, Espagne centrale).

\section{SUMMARY}

Description of a new, unpigmented and eyeless Asellid, belonging to the Iberian Genus Bragasellus Henry et Magniez, 1968.

The new species lives in the underground stream of the Cueva del Tornero (Checa, province of Guadalajara, central Spain).

\section{PLANCHE 3.}

R-Pléotelson du ơ de 7,2 mm de Bragasellus lagari $\mathrm{n} . \mathrm{sp} . ; \mathrm{e}=500$ microns.

S-Pléopode II de la 9 de $8,3 \mathrm{~mm}$, à oostégites réduits; $\mathrm{e}=200$ microns.

T-Pléopode II de la $९$ ovigère de $7,9 \mathrm{~mm} ; \mathrm{e}=200$ microns.

U-Pléopode III de la précédente; $\mathrm{e}=280$ microns.

V-Pléopode IV de la précédente; $\mathrm{e}=200$ microns.

W-Pléopode $\mathrm{V}$ de la précédente; $\mathrm{e}=200$ microns. En $\mathrm{V}$ et $\mathrm{W}$, les portions charnues des exopodites ont été pointillées. 


\section{BIBLIOGRAPHIE}

BRAGA, J.M. 1943. Description de l'Asellus seabrai, Isopode aveugle nouveau des eaux souterraines du Portugal. Mem. Est. Mus. Zool. Univ. Coimbra, 145, $1-12$.

BRAGA, J.M. 1944. Sur un Asellus nouveau des eaux superficielles du Portugal. Mem. Est. Mus. Zool. Univ. Coimbra, 154, $1-11$.

BRAGA, J.M. 1946. Quelques Asellides nouveaux du Portugal. Mem. Est. Mus. Zool. Univ. Coimbra, 173, $1-25$.

BRAGA, J.M. 1958. Un Asellus remarquable des eaux souterraines du Portugal (Asellus pauloae n.sp.), Mem. Est. Mus. Zool. Univ. Coimbra, 254, $1-15$.

BRAGA, J.M. 1964. Contribution à la faune d'Asellides du Portugal: Asellus frontellum 1964. Ann. Fac. Cienc. Porto, 46, 1-20.

HENRY, J-P. \& MAGNIEZ, G. 1970. Contribution à la systématique des Asellides (Crustacea Isopoda), Ann. Spéléol. Paris, 25,2,335 - 367.

MAGNIEZ, G. 1971. Les stations de Stenasellus virei Dollfus (Crustacé Isopode troglobie), (suite). Sous le Plancher, Dijon, 10,1, 13 - 24. 